\title{
Iodine adsorption in tetrathiafulvalene-based covalent organic frameworks
}

\author{
Jianhong Chang, ${ }^{1 \#}$ Hui Li, ${ }^{1 \#}$ Xinyu Guan, ${ }^{1}$ Cuimei Li, ${ }^{2}$ Guangtao Yu,, 2 Valentin Valtchev, ${ }^{4,5}$ Yushan \\ Yan, ${ }^{6}$ Shilun Qiu ${ }^{1}$ and Qianrong Fang ${ }^{1 *}$
}

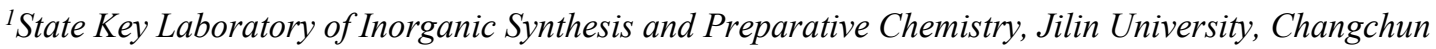
130012,P.R. China.E-mail:qrfang@jlu.edu.cn

${ }^{2}$ Laboratory of Theoretical and Computational Chemistry, Institute of Theoretical Chemistry, Jilin University, Changchun 130023, P. R. China

${ }^{3}$ College of Chemistry and Materials Science, Fujian Normal University, Fuzhou 350007, P. R. China

${ }^{4}$ Qingdao Institute of Bioenergy and Bioprocess Technology, Chinese Academy of Sciences, 189 Songling Road, Laoshan District, Qingdao, Shandong 266101, P. R. China

${ }^{5}$ Normandie Université, ENSICAEN, UNICAEN, CNRS, Laboratoire Catalyse et Spectrochimie, 14000 Caen, France

${ }^{6}$ Department of Chemical and Biomolecular Engineering, Center for Catalytic Science and Technology, University of Delaware, Newark, DE 19716, USA

${ }^{\#} J$.C. and H.L. contributed equally to this work 


\section{Abstract:}

To safeguard the development of nuclear energy, practical techniques for capture and storage of radioiodine are of critical importance but remains a significant challenge. Here we report the synergistic effect of physical and chemical adsorption of iodine in tetrathiafulvalene-based covalent organic frameworks (COFs), which can markedly improve both iodine adsorption capacity and adsorption kinetics due to their strong interaction. These functionalized architectures are designed to have high specific surface areas (up to $2359 \mathrm{~m}^{2} \mathrm{~g}^{-1}$ ) for efficient physisorption of iodine, and abundant tetrathiafulvalene functional groups for strong chemisorption of iodine. We demonstrate that these frameworks achieve excellent iodine adsorption capacity $\left(\sim 8.15 \mathrm{~g} \mathrm{~g}^{-1}\right)$ and adsorption kinetics $(\sim 0.69 \mathrm{~g}$ $\mathrm{g}^{-1} \mathrm{~h}^{-1}$ ), which are much higher than other materials reported so far, including silver-doped adsorbents, inorganic porous materials, metal-organic frameworks, porous organic frameworks, and other COFs. Furthermore, a combined theoretical and experimental study, including DFT calculations, electron paramagnetic resonance spectroscopy, X-ray photoelectron spectroscopy, and Raman spectroscopy, reveals the strong chemical interaction between iodine and framework of material. Our study thus opens an avenue to construct functional COFs for a critical environment-related application. 
Nuclear power is one of the most important energy sources due to its high power density and low carbon emission. ${ }^{1,2}$ However, an urgent safety issue related to nuclear power is the administration of waste produced from nuclear fission. ${ }^{3,4}$ Radioiodine $\left({ }^{129} \mathrm{I}\right.$ and $\left.{ }^{131} \mathrm{I}\right)$ is a typical pollutant that accompanies nuclear fission, and attracts a particular attention since it can disperse rapidly in air and causes a radiological environment up to ten million years (15.7 million year for half-life of $\left.{ }^{129} \mathrm{I}\right) .{ }^{5}$ For example, radioactive iodine leaked out from Chernobyl nuclear accident has been incorporated into the human metabolic system and resulted in human diseases like thyroid cancer. To safeguard the future development of atomic energy, efficient strategies for adsorption and removal of radioiodine are of necessary but remain elusive.

The traditional technology to remove radioactive iodine is based on a chemical transformation, that is, using silver-doped adsorbents to produce harmless AgI; however, these materials show a low efficiency. For instance, their theoretical and practical adsorption capacity is $1.18 \mathrm{~g} \mathrm{~g}^{-1}$ and $0.10-0.31 \mathrm{~g}$ $\mathrm{g}^{-1}$ (per gram Ag), respectively, which is far to face the practical needs. ${ }^{6}$ In recent years, porous materials including inorganic porous materials, ${ }^{7,8}$ metal-organic frameworks (MOFs), ${ }^{9,10}$ and porous organic frameworks (POFs), ${ }^{11,12}$ have been shown to be a suitable choice for the removal of radioiodine. In particular, covalent organic frameworks $(\mathrm{COFs}),{ }^{13-17}$ as a unique class of crystalline organic polymers with ordered porous channels, have been studied extensively for the uptake of iodine. These COF materials with specific pore environments and tunable chemistry can be easily functionalized to obtain active sites for more efficient iodine capture by electrostatic forces, Lewis acid-base interactions and coordinative sites. Nevertheless, most studies are still limited to exploitation of physical interaction, i.e., iodine uptake capacity in porous materials is driven by pore volume and uptake kinetics is dominated by pore connectivity and size. For example, although a state-of-the-art COF, TPB-DMTP COF, has achieved 
remarkable iodine adsorption capacity $\left(6.26 \mathrm{~g} \mathrm{~g}^{-1}\right)$, its adsorption kinetics is quite slow $\left(0.13 \mathrm{~g} \mathrm{~g}^{-1} \mathrm{~h}^{-1}\right) .^{18}$ In principle, the combination of high physisorption and strong chemical interaction in a material will be beneficial to improving both iodine adsorption capacity and adsorption kinetics. However, this avenue is still not properly explored.

Herein, we report the synergistic effect of physical and chemical iodine adsorption in tetrathiafulvalene (TTF)-based COFs, JUC-560 (JUC = Jilin University China) and JUC-561, leading to ultrahigh iodine adsorption capacity and ultrafast adsorption kinetics. These architectures are designed to have large specific surface areas (1815 $\mathrm{m}^{2} \mathrm{~g}^{-1}$ for JUC-560 and $2359 \mathrm{~m}^{2} \mathrm{~g}^{-1}$ for JUC-561) for high iodine uptakes through the physical process, and plentiful TTF functional groups for powerful iodine chemisorption. As a result, JUC-561 exhibits an unprecedented iodine uptake of $8.15 \mathrm{~g} \mathrm{~g}^{-1}$ and adsorption kinetics of $0.69 \mathrm{~g} \mathrm{~g}^{-1} \mathrm{~h}^{-1}$ at $333 \mathrm{~K}$ under ambient pressure. These values are much higher than other materials reported so far, including silver-doped adsorbents, inorganic porous materials, MOFs, POFs, and COFs. The strong interaction between the COF framework and iodine has been probed by DFT calculations on model molecules, $\mathrm{TTF} \cdot \mathrm{I}_{2}, \mathrm{TTF}^{+} \cdot \mathrm{I}_{2}, \mathrm{TTF}^{+} \cdot\left(\mathrm{I}^{-}\right)_{2}, \mathrm{TTF}^{+} \cdot\left(\mathrm{I}_{3}{ }^{-}\right)$, and $\mathrm{TTF}^{+} \cdot\left(\mathrm{I}_{5}{ }^{-}\right)$, and confirmed by experimental studies including X-ray photoelectron spectroscopy (XPS), electron paramagnetic resonance (EPR) spectroscopy, and Raman spectroscopy.

\section{Designed synthesis and structural analysis}

To achieve high iodine adsorption, we introduced a TTF-based derivative, tetrathiafulvalenetetrabenzaldehyde (TTF-TBA, Fig. 1b), as a planar 4-connected building unit. TTF or TTF-based derivatives are known to form the radical cations with electron acceptors (e.g., $\left.\mathrm{I}_{2}\right) .{ }^{19}$ Therefore, by the incorporation of TTF-based units, the resulting COF materials will promote iodine adsorption based on 
this strong interaction. We further chose two typical monomers, 2',5'-dimethyl-[1,1':4',1"-terphenyl]4,4"-diamine (DTDA, Fig. 1a) as a linear building unit and 2,4,6-tris(4-aminophenyl)amine (TAPA, Fig. 1c) as a 3-connected building unit. Thus, we developed two novel structures, two-dimensional (2D) JUC560 with sql topology ${ }^{20}$ and three-dimensional (3D) JUC-561 with ffc topology (Fig. 1d-i),${ }^{21}$ constructed from the condensation of TTF-TBA and linear DTDA or non-coplanar 3-connected TAPA (Supplementary Fig. 1), respectively.

The targeted COFs were synthesized by suspending TFP-TTF and DTDA or TAPT in a mixture of mesitylene/1,4-dioxane or $o$-dicholobenzene/ $n$-butanol in the presence of acetic acid, followed by heating at $120^{\circ} \mathrm{C}$ for 3 days. JUC-560 shows powder X-ray diffraction (PXRD) peaks at $2.69^{\circ}, 3.48^{\circ}$, $5.38^{\circ}, 5.60^{\circ}$, and $8.07^{\circ}$ for $2 \theta$, which are assigned to the (110), (200), (220), (310), and (330) facets, respectively. This structure adopts a 2D sql net with an eclipsed AA-stacking mode that yields a PXRD pattern similar to the experimentally observed one (Fig. 2a and Supplementary Figs. 10-12). On the other hand, JUC-561 exhibits PXRD peaks at $1.99^{\circ}, 3.21^{\circ}, 4.01^{\circ}, 5.20^{\circ}, 6.06^{\circ}, 6.89^{\circ}, 7.77^{\circ}, 8.44^{\circ}$, and $22.00^{\circ}$ for $2 \theta$, which correspond to the (020), (001), (040), (041), (060), (061), (042), (110), and (313) facets, respectively. JUC-561 assumes a 3D ffc net that exhibits a PXRD profile that consistent with the experimental one (Fig. 2b and Supplementary Figs. 13-16). PXRD patterns based on Pawley refinements $^{22}$ also confirm the assignments of PXRD peaks, as evidenced by their negligible differences (residuals $R \mathrm{p}=1.31 \%, R \mathrm{wp}=2.53 \%$ for JUC-560, and residuals $R \mathrm{p}=3.07 \%, R \mathrm{wp}=4.51 \%$ for JUC561). Based on these results, both TTF-based COFs have mesoporous frameworks with a diameter of about $2.62 \mathrm{~nm}$ for JUC-560 and about $2.55 \mathrm{~nm}$ for JUC-561 (Fig. 1f and 1g).

\section{Thermal and chemical stability}


Both COFs were observed to be chemically stable in different organic solvents, including acetone, ethanol, cyclohexanone, hexane, tetrahydrofuran, $N, N$-dimethylformamide and dimethylsulfoxide. After being treated in these solvents for $24 \mathrm{~h}$, JUC-560 and JUC-561 maintained their crystallinity (Supplementary Figs. 17 and 18). Furthermore, both COFs retained their structures in boiling water $\left(100{ }^{\circ} \mathrm{C}\right)$ and even in strong acid $\left(3 \mathrm{M} \mathrm{HCl}, 25^{\circ} \mathrm{C}\right)$ and strong base $\left(3 \mathrm{M} \mathrm{NaOH}, 25{ }^{\circ} \mathrm{C}\right)$ for $24 \mathrm{~h}$ (Supplementary Figs. 19 and 20). According to the thermogravimetric analysis (TGA), these COFs did not decompose until $400{ }^{\circ} \mathrm{C}$ under nitrogen (Supplementary Figs. 21 and 22). Therefore, these TTFbased COFs are chemically and thermally stable, crystalline, porous materials.

\section{Analysis of nitrogen gas adsorption}

The porosities of both COFs were determined by $\mathrm{N}_{2}$ adsorption and desorption isotherms at $77 \mathrm{~K}$ (Fig.

$2 \mathrm{c}$ and $2 \mathrm{~d}$ ). For each COF, a sharp uptake below $\mathrm{P} / \mathrm{P}_{0}=0.05$ and a step, indicating the presence of mesopores, was observed. The inclination of isotherms between $\mathrm{P} / \mathrm{P}_{0}=0.9-1.0$ and slight desorption hysteresis is related to the presence of textural mesopores from the agglomeration of COF crystals. ${ }^{23}$ The Brunauer-Emmett-Teller (BET) specific surface areas were calculated to be $1815 \mathrm{~m}^{2} \mathrm{~g}^{-1}$ for JUC-560 and $2359 \mathrm{~m}^{2} \mathrm{~g}^{-1}$ for JUC-561, respectively (Supplementary Figs. 23-26). The pore size distribution obtained by the nonlocal density functional theory (NLDFT) showed monomodal mesopores, $2.50 \mathrm{~nm}$ for JUC-560 and $2.46 \mathrm{~nm}$ for JUC-561 (Fig. 2e and 2f), which are in good agreement with those of the proposed model (2.62 nm for JUC-560 and $2.55 \mathrm{~nm}$ for JUC-561). Furthermore, the pore volume was determined to be $1.11 \mathrm{~cm}^{3} \mathrm{~g}^{-1}$ for JUC-560 and $1.92 \mathrm{~cm}^{3} \mathrm{~g}^{-1}$ for JUC-561, matching well with the simulated ones $\left(1.16 \mathrm{~cm}^{3} \mathrm{~g}^{-1}\right.$ for JUC-560 and $2.04 \mathrm{~cm}^{3} \mathrm{~g}^{-1}$ for JUC-561).

\section{Analysis of iodine adsorption}


We conducted iodine adsorption experiment by exposing COFs to iodine vapor at $60{ }^{\circ} \mathrm{C}$ under ambient pressure. JUC-560 exhibited a quick iodine uptake, showing a nearly linear increment in $7 \mathrm{~h}$ and then reaching adsorption saturation within $14 \mathrm{~h}$ (Fig. 3a). Similarly, JUC-561 showed a rapid adsorption in 8 $\mathrm{h}$ and achieved saturation in $18 \mathrm{~h}$ (Fig. 3b). JUC-560 obtained iodine uptakes as high as $5.17 \mathrm{~g} \mathrm{~g}^{-1}$. Remarkably, JUC-561 showed much higher iodine uptakes of $8.15 \mathrm{~g} \mathrm{~g}^{-1}$ due to its 3D framework with higher specific surface areas and interconnected channels. On the basis of the pore volume together with the iodine density $\left(4.93 \mathrm{~g} \mathrm{~cm}^{-3}\right)$, their theoretical capacities $\left(4.93 \mathrm{~g} \mathrm{~cm}^{-3} \times\right.$ pore volume) upon the full occupation of pores by iodine are $5.47 \mathrm{~g} \mathrm{~g}^{-1}$ for JUC-560 and $9.47 \mathrm{~g} \mathrm{~g}^{-1}$ for JUC-561 (Supplementary Table 1). The channels of both COFs are almost entirely occupied by iodine ( $95 \%$ for JUC-560 and $86 \%$ for JUC-561 based on their theoretical maxima), which is consistent with the high weight lost (Supplementary Figs. 27 and 28) for iodine-loading samples.

We summarized the iodine uptakes of typical adsorbents in Fig. 4 and Supplementary Table 2. As can be seen, the adsorption capacity of JUC-561 $\left(8.15 \mathrm{~g} \mathrm{~g}^{-1}\right)$ is far superior to those reported previously, such as 29-fold higher than that of the typical silver-doped zeolite mordenite (Ag-MOR, $0.28 \mathrm{~g} \mathrm{~g}^{-1}$ ), ${ }^{24}$ two orders of magnitude higher than those of nonporous materials, and much better to those of representative porous materials, including zeolitic imidazolate framework-8 (ZIF-8, $\left.1.20 \mathrm{~g} \mathrm{~g}^{-1}\right)$, ${ }^{25}$ porous aromatic framework 24 (PAF-24, $2.76 \mathrm{~g} \mathrm{~g}^{-1}$ ), ${ }^{26}$ azo-bridged porphyrin-phthalocyanine network (AzoPPN, $\left.2.90 \mathrm{~g} \mathrm{~g}^{-1}\right),{ }^{27}$ covalent organic polymers $\left(\mathrm{COP}_{1}{ }^{0}, 3.80 \mathrm{~g} \mathrm{~g}^{-1}\right),{ }^{28}$ and a state-of-the-art $\mathrm{COF}$ (TPB-DMTP COF, $\left.6.26 \mathrm{~g} \mathrm{~g}^{-1}\right) \cdot{ }^{18}$ Notably, although the specific surface area of JUC-561 $\left(2358 \mathrm{~m}^{2} \mathrm{~g}^{-1}\right)$ is lower than that of some of reported porous materials, such as COF-320 $\left(2400 \mathrm{~m}^{2} \mathrm{~g}^{-1}\right)$, NiP-conjugated microporous polymer (NiP-CMP, $2630 \mathrm{~m}^{2} \mathrm{~g}^{-1}$ ), and porous carbon (Uassis-PC800, $3053 \mathrm{~m}^{2} \mathrm{~g}^{-1}$ ), its iodine adsorption is higher (COF-320, $4.00 \mathrm{~g} \mathrm{~g}^{-1} ;{ }^{29} \mathrm{NiP}-\mathrm{CMP}, 2.02 \mathrm{~g} \mathrm{~g}^{-1.30}$ and Uassis-PC800, $2.25 \mathrm{~g}$ 
$\left.\mathrm{g}^{-1}\right)^{31}$ due to the synergistic effect of physical and chemical adsorption of iodine in JUC-561.

The adsorption kinetics of iodine in various materials were also summarized in Supplementary Table S3. In fact, the available relevant reports are limited due to low adsorption kinetics in most of previously reported materials. Remarkably, the adsorption kinetics of TTF-based COFs $\left(0.48 \mathrm{~g} \mathrm{~g}^{-1} \mathrm{~h}^{-1}\right.$ for JUC-560 and $0.69 \mathrm{~g} \mathrm{~g}^{-1} \mathrm{~h}^{-1}$ for JUC-561) is superior to those adsorbents reported, such as nitrogen-rich triptycenebased porous polymer (NTP, $\left.0.11 \mathrm{~g} \mathrm{~g}^{-1} \mathrm{~h}^{-1}\right),{ }^{32}$ TTA-TTB COF $\left(0.15 \mathrm{~g} \mathrm{~g}^{-1} \mathrm{~h}^{-1}\right),{ }^{18} \mathrm{NiP}-\mathrm{CMP}\left(0.17 \mathrm{~g} \mathrm{~g}^{-1} \mathrm{~h}^{-}\right.$ $\left.{ }^{1}\right),{ }^{30} \mathrm{AzoPPN}\left(0.18 \mathrm{~g} \mathrm{~g}^{-1} \mathrm{~h}^{-1}\right),{ }^{27}$ and even more than 34 times higher than that $\left(0.02 \mathrm{~g} \mathrm{~g}^{-1} \mathrm{~h}^{-1}\right)$ of Ag-loaded zeolites applied in real environment. ${ }^{24}$ These results confirm that the strong physical and chemical interaction between TTF units and iodine is favorable to enhance iodine adsorption ability.

The iodine-adsorbed COFs can preserve iodine upon exposure to air under ambient conditions $\left(25^{\circ} \mathrm{C}\right.$ and 1 bar), and do not display iodine escape from the frameworks (Fig. 3c and 3d). Adsorbed iodine could be removed by immersing these samples in ethanol solution at room temperature. Iodine release was monitored by optical images and UV/Vis spectroscopy, leading to a linear increase based on the absorbance over time (Supplementary Figs. 29-36). After ethanol rinse, these COFs were recyclable and retained similar iodine uptakes (Fig. 3e and 3f). FT-IR spectroscopy verified the chemical integrity of these COFs after cycled application (Supplementary Figs. 37 and 38). These regenerated COFs maintained their crystallinity and porosity, as evidenced by their unchanged PXRD patterns and retained specific surface areas (Supplementary Figs. 39-42). These excellent cycle performances suggest that these materials are robust against oxidative iodine over long-term exposure.

\section{Experimental study of the interaction between iodine and framework}

Electron paramagnetic resonance (EPR) studies of the solid samples at $110 \mathrm{~K}$ confirmed the 
generation of TTF radical cations after iodine adsorption (Fig. 5a and 5b). Clearly, the pristine samples showed a very weak EPR signal (black curve) while there is an approximately two orders of magnitude increase in paramagnetic intensity for the iodine-doped samples (red curve). After $\mathrm{I}_{2}$ doping, sharp peaks were observed at $\mathrm{g}=2.0182$ for JUC-560 and 2.0189 for JUC-561 respectively, clearly demonstrating the presence of TTF radical cations from oxidation by iodine. ${ }^{33}$ Furthermore, we tested the electrochemical behavior of JUC-560 and JUC-561 by cyclic voltammetry (CV). In Supplementary Figs. 43 and 44, two clear redox processes at 0.72 and $1.15 \mathrm{~V}$ vs Ag/AgCl for JUC-560 and 0.69 and $1.08 \mathrm{~V}$ vs $\mathrm{Ag} / \mathrm{AgCl}$ for JUC-561 were observed in the reversible CV profiles, revealing that TTF units retain their redox activities in the frameworks and can be oxidized by iodine which has a reduction potential at $0.78 \mathrm{~V}^{34}$

To investigate the existing state of iodine captured in TTF-based COFs, we conducted X-ray photoelectron spectroscopy (XPS). In Fig. 5c and 5d, two conspicuous peaks located at $629.8 \mathrm{eV}$ and $618.3 \mathrm{eV}$ for JUC-560 as well as $630.0 \mathrm{eV}$ and $618.6 \mathrm{eV}$ for JUC-561, belonging to I $3 \mathrm{~d}_{3 / 2}$ and $\mathrm{I} 3 \mathrm{~d}_{5 / 2}$ orbital of iodine molecules respectively, were observed, which indicates that the captured iodine partially exists as a molecule. Furthermore, another two peaks at $630.9 \mathrm{eV}$ and $619.6 \mathrm{eV}$ for JUC-560 as well as $631.4 \mathrm{eV}$ and $619.8 \mathrm{eV}$ for JUC-561 were found, which are attributed to the formation of polyiodine anions (such as $\mathrm{I}_{3}^{-}$and $\mathrm{I}_{5}^{-}$). ${ }^{35}$ In addition, the species of captured iodine were detected by Raman spectroscopy. As shown in Fig. 5e and 5f, the Raman spectra of TTF-based COFs showed no distinct peaks before iodine adsorption (black curve). However, after iodine capture, the low-frequency spectral region was dominated by three peaks at 107, 136 and $174 \mathrm{~cm}^{-1}$ for JUC-560 and 108, 135 and $181 \mathrm{~cm}^{-1}$ for JUC-561 (red curve). The peaks can be assigned to the symmetric and asymmetric stretching vibrations of $\mathrm{I}_{3}{ }^{-}$as well as the stretching vibrations of $\mathrm{I}_{5}{ }^{-}$, respectively. ${ }^{36}$ Thus the results show that the 
adsorbed iodine species in TTF-based COFs exist as both iodine molecule and polyiodine anions, and the process is the combination of physisorption and chemisorption.

\section{Theoretical study of the interaction between iodine and framework}

To gain insight into how the COF frameworks bind iodine, we performed DFT calculations with the M06-2X-D3 function based on Gaussian 09 package (Revision D.01). ${ }^{37}$ Full geometry optimizations of model compounds, TTF $\cdot \mathrm{I}_{2}, \mathrm{TTF}^{+} \cdot \mathrm{I}_{2}, \mathrm{TTF}^{+\cdot} \cdot\left(\mathrm{I}^{-}\right), \mathrm{TTF}^{++} \cdot\left(\mathrm{I}_{3}{ }^{-}\right)$, and $\mathrm{TTF}^{+\cdot} \cdot\left(\mathrm{I}_{5}{ }^{-}\right)$, offered related systems with the $\mathrm{I}_{2}$ molecule, $\mathrm{I}^{-}, \mathrm{I}_{3}{ }^{-}$or $\mathrm{I}_{5}{ }^{-}$anion situating on the center of TTF or $\mathrm{TTF}^{+}$unit (Supplementary Fig. 45 ). No symmetry constraints were imposed during the optimizations. The standard def2-SVP basis set was used for geometry optimizations, and the vibration analysis and def2-TZVP basis set was applied for calculating single point energy. The binding energy of $\mathrm{I}_{2}$ molecule to the TTF or $\mathrm{TTF}^{+}$unit calculated at the M06-2X-D3 level was low (-8.3 kcal mol-1 and $-7.1 \mathrm{kcal} \mathrm{mol}^{-1}$ respectively). However, the association of $\mathrm{I}^{-}$anion with the $\mathrm{TTF}^{+}$unit showed a large binding energy as high as $-74.6 \mathrm{kcal} \mathrm{mol}^{-1}$, which is the result of the electrostatic interaction between the anions and the positively charged viologen. ${ }^{38}$ Similarly, the $\mathrm{I}_{3}{ }^{-}$or $\mathrm{I}_{5}{ }^{-}$anion also displayed a relatively high binding energy of $-70.6 \mathrm{kcal} \mathrm{mol}^{-1}$ or $-65.3 \mathrm{kcal} \mathrm{mol}^{-1}$ to $\mathrm{TTF}^{+}$unit, respectively. Thus, these results suggest that TTF molecule reducing to its diradical form leads to the change of $\mathrm{I}_{2}$ to $\mathrm{I}^{-}, \mathrm{I}_{3}{ }^{-}$or $\mathrm{I}_{5}^{-}$anion, which at last is tightly bound to the framework of COFs through electrostatic interactions.

\section{Outlook}

By developing TTF-based COF materials, we have successfully obtained high iodine adsorption capacity and quick iodine adsorption kinetics. These results point out that the integration of virtues of physical and chemical adsorption is of paramount importance in the designed synthesis of porous 
materials for the very challenging capture of radioiodine. The pre-designed COF frameworks exhibiting synergy between physisorption and chemisorption showed a dual impact on the iodine adsorption: substantially improved total iodine capacity, in particular the 3D COF (JUC-561); and accelerated transport rate due to their strong interaction between functionalized units and iodine. Consequently, the TTF-based COFs achieved the highest iodine uptake $\left(\sim 8.15 \mathrm{~g} \mathrm{~g}^{-1}\right)$ and adsorption kinetics $\left(\sim 0.69 \mathrm{~g} \mathrm{~g}^{-1}\right.$ $\mathrm{h}^{-1}$ ), which are far superior to other materials reported so far, such as silver-doped adsorbents, inorganic porous materials, MOFs, POFs, and other COFs. The set of experimental results thus demonstrate the potential of functionalized COF materials as a platform for radioiodine capture and storage.

Some prospective directions for future work can be exploited to obtain efficient iodine adsorption. COF materials feature open pores and excellent specific surface areas, and thus hold great promises in iodine adsorption. Unlike 2D structures with characteristic eclipsed AA stacking, 3D COFs have attracted widespread attentions owing to their interconnected channels and impressive specific surface areas. Therefore, the development of 3D COFs with different architectures are interesting for iodine capture due to their unique pore shape and pore environment. In addition, COFs are well-defined in which their organic building units are precisely integrated into extended structures, and thus they can be easily functionalized to obtain strong chemisorption of iodine. A number of functionalized groups as electron donors, such as carbazole and ionic liquid, can be decorated into the frameworks by bottom-up or postsynthetic approaches. Most importantly, by the integration of higher specific surface areas and abundant functional units, we predict that the iodine adsorption in COF materials could be further improved. These functionalized porous frameworks would be applicable in environmental protection for the capture of radioiodine and other hazardous substances.

\section{References:}


1. Kintisch, E. Congress tells DOE to take fresh look at recycling spent reactor fuel. Science. 310, 1406$1406(2005)$.

2. Vienna, J. D. Nuclear waste vitrification in the United States: recent developments and future options. Int. J. Appl. Glass Sci. 1, 309-321 (2010).

3. Ewing, R. C. \& Hippel, F. N. von. Nuclear waste management in the United States-starting over. Science 325, 151-152 (2009).

4. Soelberg, N. R. et al. Radioactive iodine and krypton control for nuclear fuel reprocessing facilities. Sci. Technol. Nucl. Install. 2013, 702496 (2013).

5. Ojovan, M. I. \& Lee, W. E. An introduction to nuclear waste immobilization, Elsevier Science Amsterdam. 2005.

6. Riley, B. J. Vienna, J. D. Strachan, D. M. McCloy, J. S. \& Jerden, J. L. Materials and processes for the effective capture and immobilization of radioiodine: A review. J. Nucl. Mater. 470, 307-326 (2016).

7. Hertzsch, T. Budde, F. Weber, E. \& Hülliger, J. Supramolecular-wire confinement of $I_{2}$ molecules in channels of the organic zeolite tris(o-phenylenedioxy)cyclotriphosphazene. Angew. Chem. Int. Ed. 41, 2281-2284 (2002).

8. Mohanambe, L. \& Vasudevan, S. Insertion of iodine in a functionalized inorganic layered solid. Inorg. Chem. 43, 6421-6425 (2004).

9. Long, J. R. \& Yaghi, O. M. The pervasive chemistry of metal-organic frameworks. Chem. Soc. Rev. 38, 1213-1213 (2009).

10. Zhou, H. C. Long, J. R. \& Yaghi, O. M. Introduction to metal-organic frameworks. Chem. Rev. 112, 
673-674 (2012).

11. Hasell, T. Schmidtmann, M. \& Cooper, A. I. Molecular doping of porous organic cages. J. Am. Chem. Soc. 133, 14920-14923 (2011).

12. Pei, C. Y. Ben, T. Xu, S. X. \& Qiu, S. L. Ultrahigh iodine adsorption in porous organic frameworks. J. Mater. Chem. A. 2, 7179-7187 (2014).

13. Côté, A. P. et al. Porous, crystalline, covalent organic frameworks. Science. 310, 1166-1170 (2005).

14. Colson, J. W. \& Dichtel, W. R. Rationally synthesized two-dimensional polymers. Nat. Chem. 5, $453-465$ (2013).

15. Ding, S. Y. \& Wang, W. Covalent organic frameworks (COFs): from design to applications. Chem. Soc. Rev. 42, 548-568 (2013).

16. Geng, K. Y. et al. Covalent organic frameworks: design, synthesis, and functions. Chem. Rev. doi.org/10.1021/acs.chemrev.9b00550.

17. Guan, X. Y., Chen, F. Q., Fang, Q. R. \& Qiu, S. L. Design and applications of three dimensional covalent organic frameworks. Chem. Soc. Rev. 49, 1357-1384 (2020).

18. Wang, P. et al. Exceptional iodine capture in 2D covalent organic frameworks. Adv. Mater. 30, 1801991 (2018).

19. Li, H. et al. Three-dimensional tetrathiafulvalene-based covalent organic frameworks for tunable electrical conductivity. J. Am. Chem. Soc. 141, 13324-13329 (2019).

20. http://rcsr.net/nets. 
21. Lan, Y. S. et al. Materials genomics methods for high-throughput construction of COFs and targeted synthesis. Nat. Commun. 9, 5274 (2018).

22. Materials Studio v.7.0 (Accelrys Inc, 2013); http://www.3dsbiovia.com/products/collaborativescience/biovia-materials-studio/

23. Fang, Q. et al. 3D porous crystalline polyimide covalent organic frameworks for drug delivery. J. Am. Chem. Soc. 137, 8352-8355 (2015).

24. Chapman, K. W. Chupas, P. J. \& Nenoff, T. M. Radioactive iodine capture in silver-containing mordenites through nanoscale silver iodide formation. J. Am. Chem. Soc. 132, 8897-8899 (2010).

25. Sava, D. F. et al. Capture of volatile iodine, a gaseous fission product, by zeolitic imidazolate framework-8. J. Am. Chem. Soc. 133, 12398-12401 (2011).

26. Yan, Z. Yuan, Y. Tian, Y. Zhang, D. \& Zhu, G. Highly efficient enrichment of volatile iodine by charged porous aromatic frameworks with three sorption sites. Angew. Chem. Int. Ed. 54, 12733-12737 (2015).

27. Li, H. Ding, X. \& Han, B. Porous azo-bridged porphyrin-phthalocyanine network with high iodine capture capability. Chem. Eur. J. 22, 11863-11868 (2016).

28. Das, G. et al. Multifunctional redox-tuned viologen-based covalent organic polymers. J. Mater. Chem. A. 4, 15361-15369 (2016).

29. An, S. et al. Porosity modulation in two-dimensional covalent organic frameworks leads to enhanced iodine adsorption performance. Ind. Eng. Chem. Res. 58, 10495-10502 (2019).

30. A, S. et al. Highly efficient and reversible iodine capture using a metalloporphyrin-based conjugated 
microporous polymer. Chem. Commun. 50, 8495-8498 (2014).

31. Xiao, K. et al. Excellent performance of porous carbon from urea-assisted hydrochar of orange peel for toluene and iodine adsorption. Chem. Eng. J. 382, 122997 (2020).

32. Ma, H. et al. Nitrogen-rich triptycene-based porous polymer for gas storage and iodine enrichment. ACS Macro. Lett. 5, 1039-1043 (2016).

33. Berridge, R. et al. Incorporation of fused tetrathiafulvalenes (TTFs) into polythiophene architectures: varying the electroactive dominance of the TTF species in hybrid systems. J. Phys. Chem. B. 110, 31403152 (2006).

34. Qi, P. H. Hiskey, J. B. Electrochemical behaviour of gold in iodide solutions. Hydrometallurgy. 32, 161-179 (1993).

35. Hsu, S. Signorelli, A. Pez, G. Baughman, R. Highly conducting iodine derivatives of polyacetylene: Raman, XPS and X-ray diffraction studies. J. Chem. Phys. 69, 106-111 (1978).

36. Harijan, D. Chandra, V. Yoon, T. Kim, K. Radioactive iodine capture and storage from water using magnetite nanoparticles encapsulated in polypyrrole. J. Hazard Mater. 344, 576-584 (2018).

37. Frisch, M. J. et al. Gaussian 09; Revision D.01, Gaussian, Inc.: Wallingford, CT (2009).

38. Das, G. Multifunctional redox-tuned viologen-based covalent organic polymers. J. Mater. Chem. A. 4, 15361 (2016).

\section{Acknowledgements}

We thank National Natural Science Foundation of China (21571079, 21621001, 21390394, 21571076, 21673094, and 21571078), "111" project (BP0719036 and B17020), the program for JLU Science and 
Technology Innovative Research Team for funding, and the Computing Center of Jilin Province for supercomputer time. We thank Prof. L. Chen for help at SEM images. We thank Prof. J. Jiang for help at EPR spectroscopy. We thank Prof. C. Hou for help at XPS spectroscopy. We thank Prof. S. Xu for help at Raman spectroscopy.

\section{Author contributions}

J.C. and H.L. performed the synthesis and characterization of COF samples and measurements of nitrogen and iodine adsorption. X.G., C.L. and G.Y. performed the DFT calculations. Q.F. led the overall design and direction of the project. Q.F., V.V., Y.Y., and S.Q. prepared the manuscript with help from all authors.

\section{Competing interests}

The authors declare no competing interests.

\section{Additional information}

Supplementary information is available for this paper.

Correspondence and requests for materials should be addressed to Q.F.

\section{Methods}

Materials. All starting materials and solvents, unless otherwise noted, were obtained from J\&K scientific

LTD. The reagents and purity of the solvents were of $95 \%$ and used without further purification.

TFP-TTF, DTDA, and TAPA were synthesized using a modified literature method. All products were isolated and handled under nitrogen using either glovebox or Schlenk line techniques.

Instruments. A Bruker AV-400 NMR spectrometer was applied to record the liquid ${ }^{1} \mathrm{H}$ NMR 
spectroscopy. Solid-state ${ }^{13} \mathrm{C}$ NMR spectroscopy was recorded on an AVIII $500 \mathrm{MHz}$ solid-state NMR spectrometer. FT-IR spectroscopy (KBr) was obtained using a SHIMADZU IRAffinity-1 Fourier transform infrared spectrophotometer. Thermogravimetric analysis (TGA) was recorded on a SHIMADZU DTG-60 thermal analyzer under $\mathrm{N}_{2}$. The operational range of the instrument was from $30{ }^{\circ} \mathrm{C}$ to $600{ }^{\circ} \mathrm{C}$ at a heating rate of $10{ }^{\circ} \mathrm{C} \mathrm{min}^{-1}$ with $\mathrm{N}_{2}$ flow rate of $30 \mathrm{~mL} \mathrm{~min}{ }^{-1}$. PXRD data were collected on a PANalytical B.V. Empyrean powder diffractometer using a $\mathrm{Cu} \mathrm{K} \alpha$ source $(\lambda=1.5418 \AA)$ over the range of $2 \theta=2.0-40.0^{\circ}$ with a step size of $0.02^{\circ}$ and $2 \mathrm{~s}$ per step. The sorption isotherm for $\mathrm{N}_{2}$ was measured by using a Quantachrome Autosorb-IQ analyzer with ultra-high-purity gas (99.999\% purity). Before gas adsorption measurements, the as-synthesized COFs ( $\sim 50.0 \mathrm{mg})$ were immersed in DMF for $12 \mathrm{~h}(3 \times 5.0 \mathrm{ml})$ and then acetone for another $36 \mathrm{~h}(3 \times 5.0 \mathrm{ml})$. The acetone was then extracted under vacuum at $85^{\circ} \mathrm{C}$ to afford the samples for sorption analysis. To estimate pore size distributions for TTF-COFs, nonlocal density functional theory (NLDFT) was applied to analyze the $\mathrm{N}_{2}$ isotherm on the basis of the model of $\mathrm{N}_{2} @ 77 \mathrm{~K}$ on carbon with slit pores and the method of non-negative regularization. For scanning electron microscopy (SEM) images, JEOL JSM-6700 scanning electron microscope was applied. The transmission electron microscopy (TEM) images were obtained on JEM-2100 transmission electron microscopy. The Electrochemistry experiments were conducted on a CHI660C Electrochemical Workstation (Shanghai Chenhua Electrochemical Instrument). Electron paramagnetic resonance (EPR) spectra were measured on JES-FA200. Raman scattering spectra were measured on INVIA, and the Raman band of the silicon wafer at $520.7 \mathrm{~cm}^{-1}$ was used to calibrate the spectrometer. X-ray photoelectron spectroscopy (XPS) measurements were performed on an ESCALAB 250 XPS system.

Synthesis of JUC-560. DTDA (0.05 mmol, $17.3 \mathrm{mg})$ and TFP-TTF (0.025 mmol, $18.6 \mathrm{mg})$ were weighted into a Pyrex tube (volume: ca. $20.0 \mathrm{ml}$ with a body length of $18.0 \mathrm{~cm}$ and neck length of 9.0 
$\mathrm{cm})$. Then, the mixture of mesitylene $(0.7 \mathrm{~mL}), 1,4$-dioxane $(0.3 \mathrm{~mL})$ and $0.1 \mathrm{ml}$ of aqueous acetic acid $(6.0 \mathrm{~mol} / \mathrm{L})$ was added. The Pyrex tube was flash-frozen in a liquid nitrogen bath and evacuated to an internal pressure of ca. 19.0 mbar and flame-sealed, reducing the total length by ca. $10.0 \mathrm{~cm}$. Upon warming to room temperature, the tube was placed in an oven at $120^{\circ} \mathrm{C}$ for three days. As a result, a pale brown powder was isolated by centrifugation and washed with acetone $(3 \times 5.0 \mathrm{ml})$ and the yield is about 83\%. Anal. Cald for $\mathrm{C}_{37} \mathrm{H}_{26} \mathrm{~N}_{2} \mathrm{~S}_{2}$ : C: 78.97; H: 4.66; N: 4.98; S: 11.39. Found: C: 79.12; H: 4.61; N: 5.06; S: 11.21. Solid-state ${ }^{13} \mathrm{C}$ NMR (500 MHz) $\delta$ (ppm): 159.4, 150.8, 141.1, 131.9, 121.2, 20.2. FT-IR: (KBr), $v / \mathrm{cm}^{-1}: 3024.24,2866.98,2733.86,2361.54,1774.92,1603.03,1555.49,1483.80,1307.52,1102.72$, $1012.02,840.13$

Synthesis of JUC-561. TAPA (0.04 mmol, $13.4 \mathrm{mg})$ and TFP-TTF $(0.03 \mathrm{mmol}, 21.7 \mathrm{mg})$ were weighted into a Pyrex tube (volume: ca. $20.0 \mathrm{ml}$ with a body length of $18.0 \mathrm{~cm}$ and neck length of $9.0 \mathrm{~cm}$ ). Then, the mixture of $o$-dicholobenzene $(0.2 \mathrm{~mL}), n$-butanol $(0.8 \mathrm{~mL})$, and aqueous acetic acid $(9.0 \mathrm{~mol} / \mathrm{L}, 0.1$ $\mathrm{mL}$ ) was added. The Pyrex tube was flash-frozen in a liquid nitrogen bath and evacuated to an internal pressure of ca. 19.0 mbar and flame-sealed, reducing the total length by ca. $10.0 \mathrm{~cm}$. Upon warming to room temperature, the tube was placed in an oven at $120^{\circ} \mathrm{C}$ for five days. As a result, a pale brown powder was isolated by centrifugation and washed with acetone $(3 \times 5.0 \mathrm{ml})$ and the yield is about $81 \%$. Anal. Cald for $\mathrm{C}_{87} \mathrm{H}_{54} \mathrm{~N}_{8} \mathrm{~S}_{6}$ : C: 74.44; H: 3.88; N: 7.98; S: 13.70. Found: C: 74.46; H: 3.85; N: 7.99; S: 13.69. Solid-state ${ }^{13} \mathrm{C}$ NMR (500 MHz) $\delta$ (ppm): 157.9, 155.4, 144.6, 135.9, 128.3. FT-IR: (KBr), $v / \mathrm{cm}^{-}$ ${ }^{1}: 3033.02,2840.64,2740.43,1902.19,1701.77,1602.56,1494.04,1309.71,1209.51,1109.29,832.81$.

General procedure for iodine vapor sorption. An open small vial $(2.0 \mathrm{~mL})$ containing the COF sample (20.0 $\mathrm{mg})$ was placed in a large vial $(10.0 \mathrm{~mL})$ containing iodine $(1.0 \mathrm{~g})$. The large vial was sealed and kept in an oven at $60{ }^{\circ} \mathrm{C}$. After a certain period, the large vial was cooled to room temperature. The small 
vial containing the COF sample was weighted and placed back into the iodine-containing large vial. The large vial was sealed and put back in the oven at $60{ }^{\circ} \mathrm{C}$ to continue the adsorption till the mass of the small vial containing the COF sample did not change.

General procedure for recycling the COF samples. The iodine-captured COF sample was added to ethanol $(20.0 \mathrm{~mL})$ in a vial at $25^{\circ} \mathrm{C}$, and ethanol was refreshed every $2 \mathrm{~h}$ until no color of the solution was observed. The COF sample was collected by filtration, washed with ethanol, dried under vacuum at $120^{\circ} \mathrm{C}$ overnight and reused for the next cycle.

General procedure for iodine escape investigation. An open vial $(2.0 \mathrm{~mL})$ containing the iodine-loaded COF sample was placed in a large empty vial $(10.0 \mathrm{~mL})$. The large vial was sealed and kept in room temperature. After a certain period, the small vial containing the COF sample was weighted and placed back into the large empty vial. The large vial was sealed to continue the desorption. The investigation was carried out for a period of 8 days.

Cyclic voltammetry (CV) measurements. After grinding each as-synthesized COF (6.0 mg) and carbon black $(4.0 \mathrm{mg})$ in agate mortar and pestle for 10 mins, the grinded powder was subjected to a mixture of water $(0.25 \mathrm{~mL})$, ethanol $(0.25 \mathrm{~mL})$ and Nafion soluion $(50.0 \mu \mathrm{L})$ and sonicated for $2 \mathrm{~h}$. Then the electrode was prepared by drop-casting such mixture onto the surface of carbon fiber which was dried before use. The $\mathrm{CV}$ measurements were performed using an electrochemical analyzer $\mathrm{CHI} 760 \mathrm{i} \mathrm{E}$ and a standard three-electrode setup with a carbon working electrode, platinum wire auxiliary electrode and $\mathrm{Ag} / \mathrm{AgCl}$ as the reference electrode. The scan rate was $20 \mathrm{mV} \cdot \mathrm{s}^{-1}$ with potential range $0 \mathrm{~V}$ to $1.5 \mathrm{~V}$ in supporting electrolyte $\left(0.1 \mathrm{M} \mathrm{NBu}_{4} \mathrm{PF}_{6}\right.$ in $\left.\mathrm{CH}_{2} \mathrm{Cl}_{2}\right)$. 
Figures and captions:

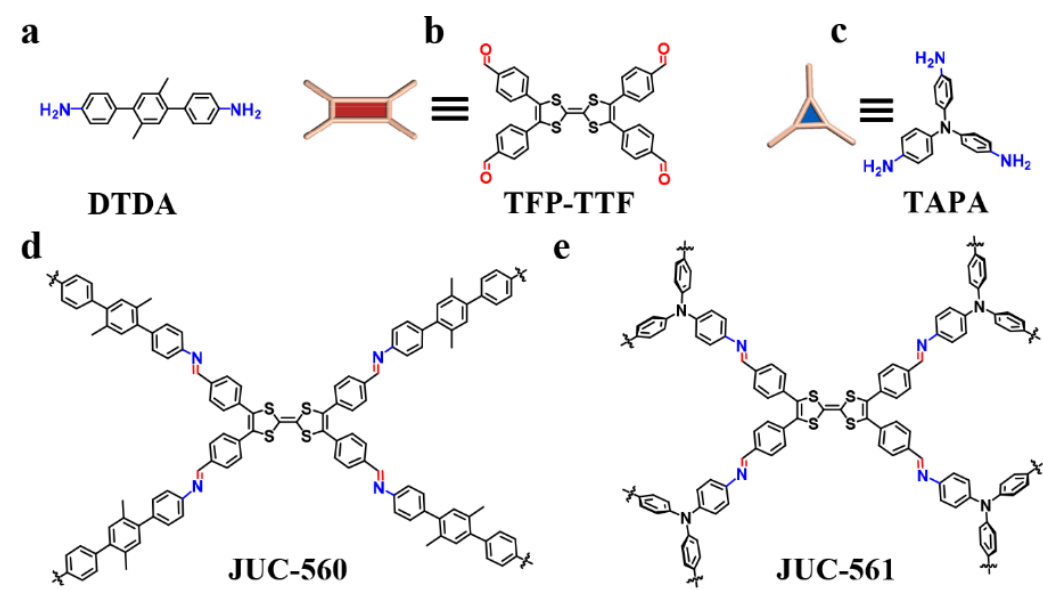

f

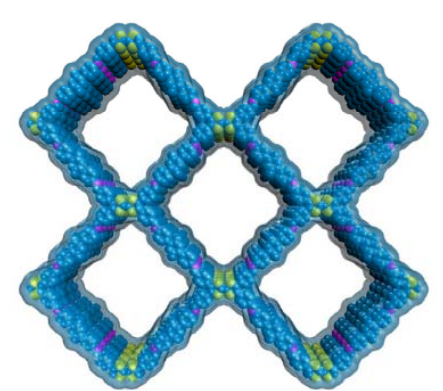

h

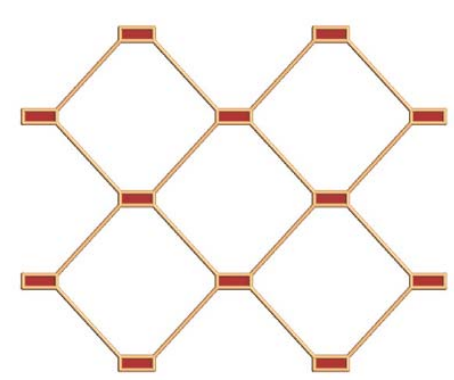

2D sql net g

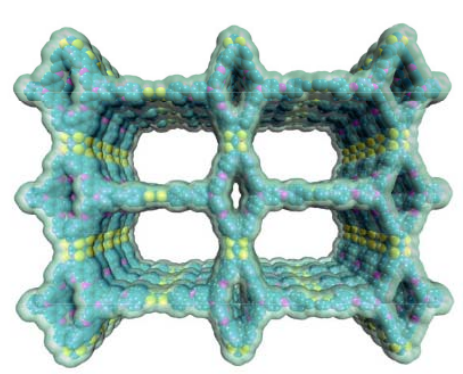

i

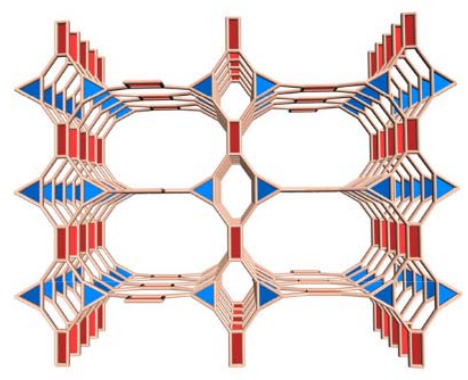

3D ffe net

Fig. 1 Schematic Representation of the Strategy for Preparing TTF-based COFs. (a-c) Molecular structures of DTDA as a linear building unit, TFP-TTF (a) as a 4-connected building unit, and TAPA (c) as a 3-connected non-coplanar building unit. (d and e) Two novel TFF-based COFs, denoted as JUC560 (d) and JUC-561 (e), are constructed by the condensation reaction of TTF and DTDA or TAPA. (f and g) Extended structures of mesoporous 2D JUC-560 (a) and 3D JUC-561 (b). (h and i) 2D sql and 3D ffc net in JUC-560 (h) and JUC-561 (i) respectively. 

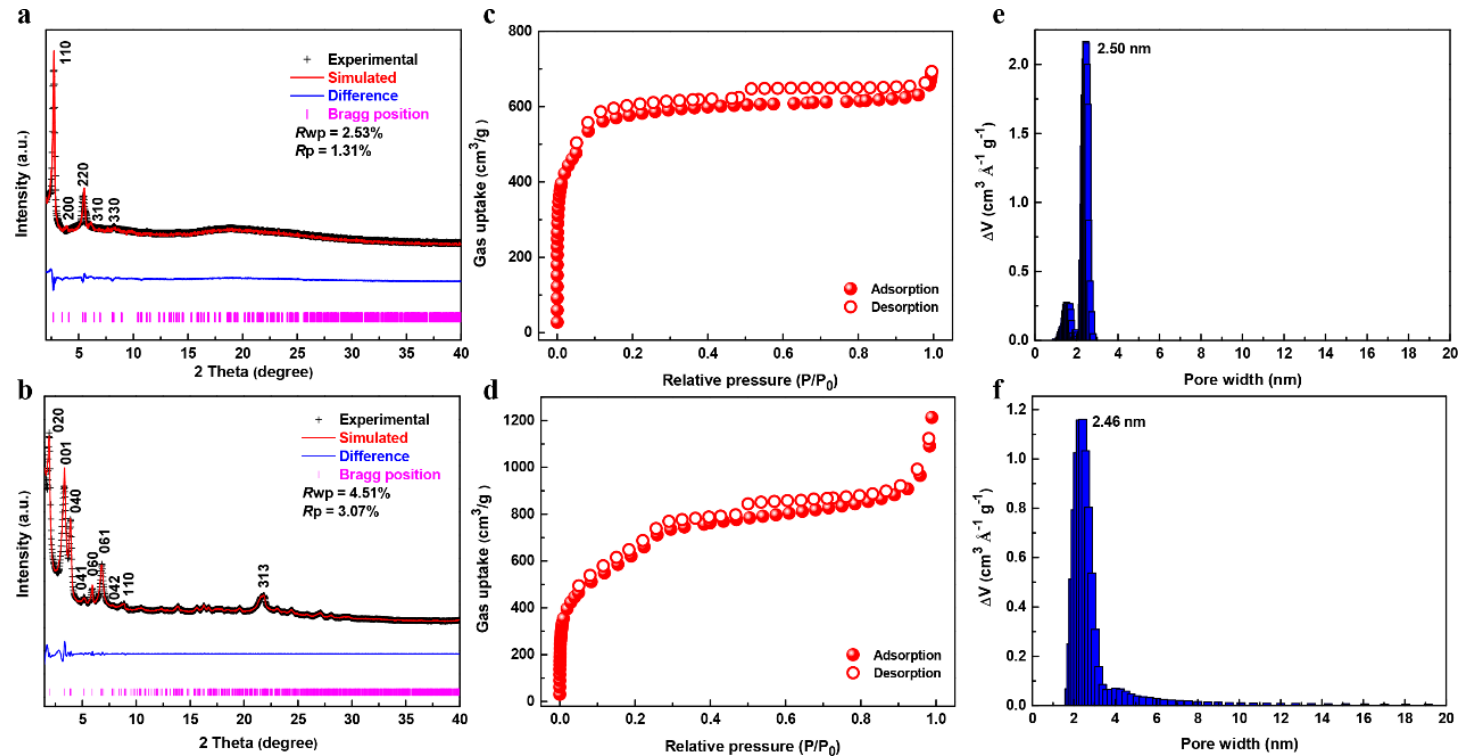

Fig. 2 PXRD profiles and porosity. (a and b) PXRD patterns of JUC-560 (a) and JUC-561 (b). (c and d) $\mathrm{N}_{2}$ adsorption-desorption isotherms for JUC-560 (c) and JUC-561 (d) at $77 \mathrm{~K}$. (e and f) Pore-size distribution of JUC-560 (e) and JUC-561 (f) calculated by fitting on the NLDFT model to the adsorption data. 


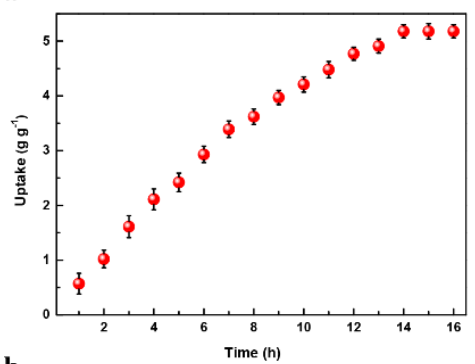

b

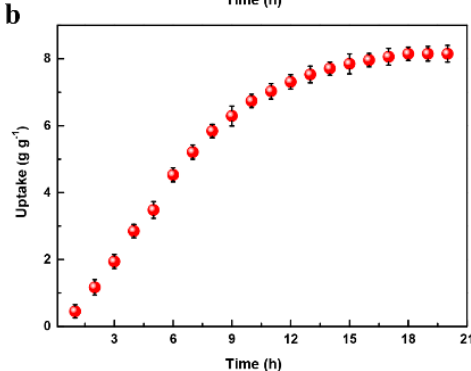

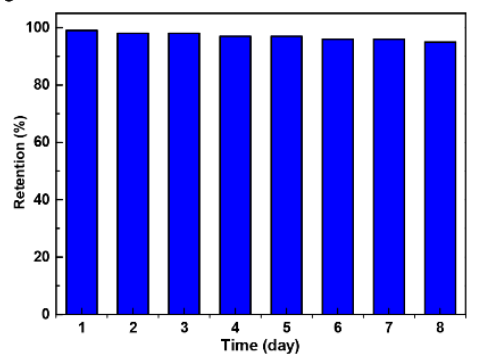

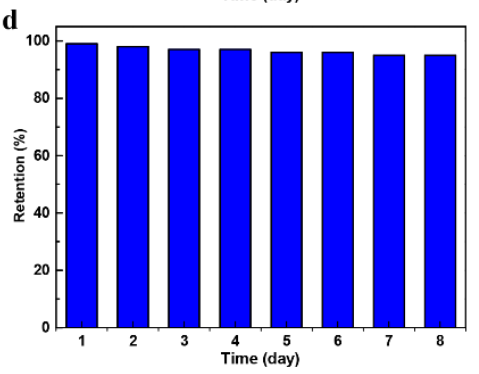

e
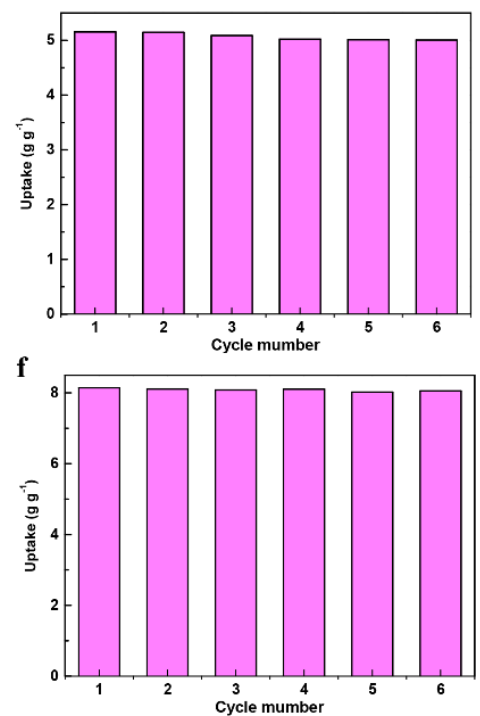

Fig. 3 Study of iodine capture. (a and b) Uptake of iodine of JUC-560 (a) and JUC-561 (b) as a function of exposure time at $338 \mathrm{~K}$ and ambient pressure. (c and d) Iodine retention of the iodine-captured JUC560 (c) and JUC-561 (d) upon exposure to air at $298 \mathrm{~K}$ and ambient pressure. (e and f) Recyclability of JUC-560 (e) and JUC-561 (f) in iodine adsorption. 


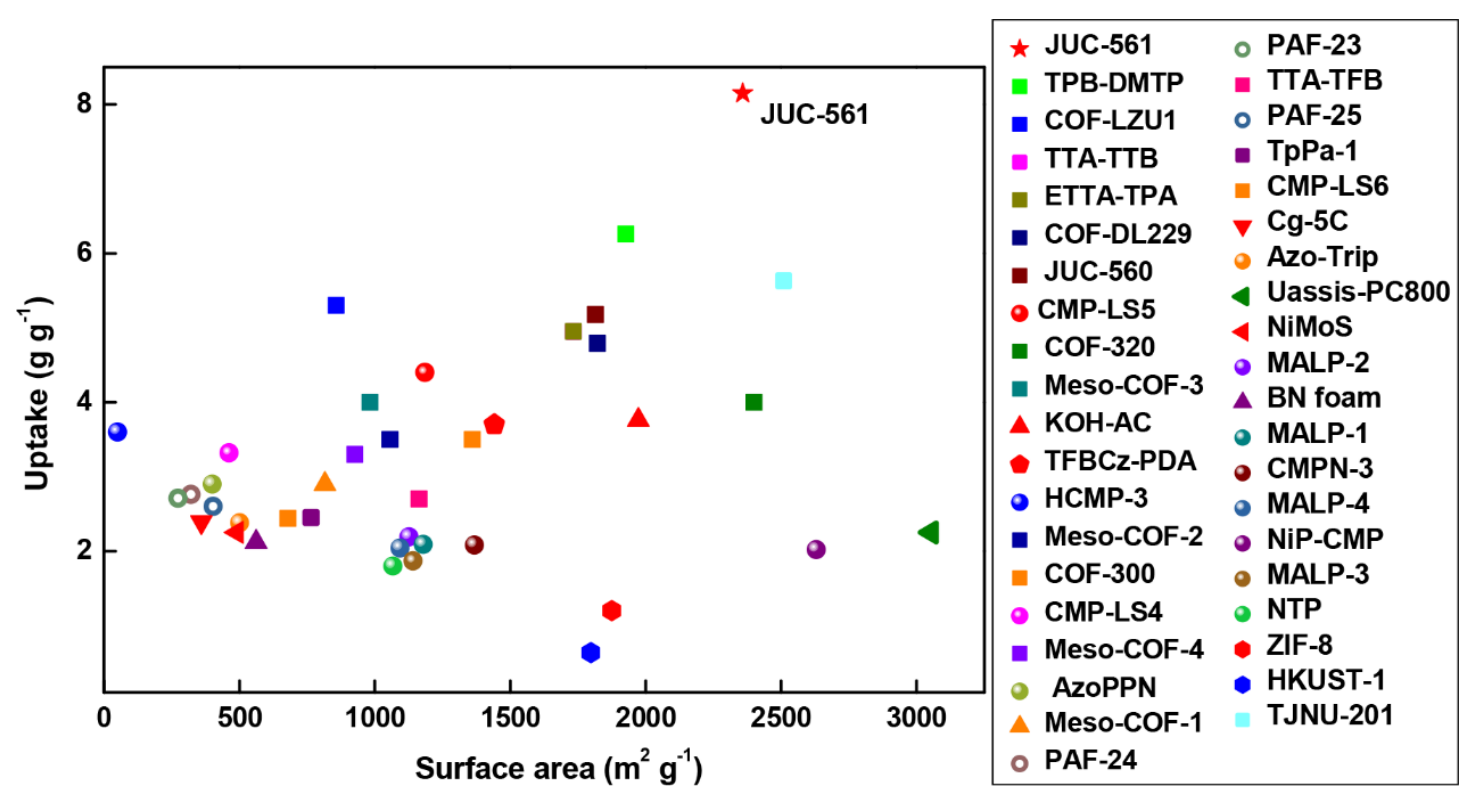

Fig. 4 Comparison of iodine adsorption capacity in different adsorbents. A plot of iodine adsorption against the specific surface area of different adsorbents. 

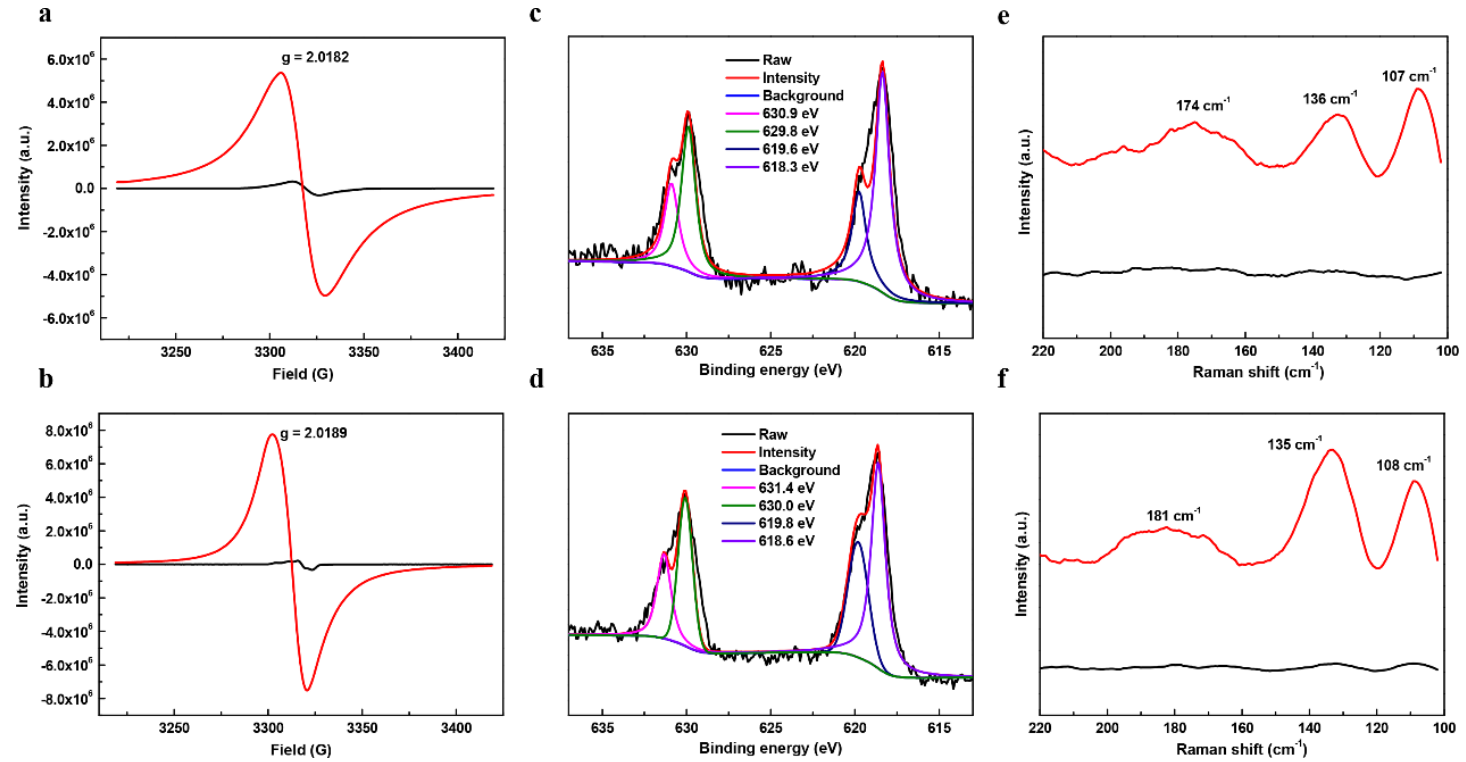

Fig. 5 Spectroscopy study. (a and b) EPR spectroscopy of JUC-560 (a) and JUC-561 (b) before (black curve) and after (red curve) iodine uptake. (c and d) XPS of iodine for JUC-560 (c) and JUC-561 (d) after iodine uptake. (e and f) Raman spectroscopy of JUC-560 (e) and JUC-561 (f) before (black curve) and after (red curve) iodine uptake. 